\title{
Shielding Effectiveness of Liquid Electrolyte
}

\author{
S. Kovar ${ }^{1}$, M. Pospisilik ${ }^{1}$, J. Valouch ${ }^{1}$, and M. Adamek ${ }^{1}$ \\ ${ }^{1}$ Faculty of Applied Informatics Tomas Bata University in Zlín, Czech Republic
}

\begin{abstract}
The paper aims at the investigation of a liquid electrolyte effect on electromagnetic radiation. The liquid electrolyte could represent an interesting solution against electromagnetic interference for devices using transparent covers, such as security cameras. Currently, the way how to protect transparent areas is a fine metallic grid or polymer material; however, this solution could reduce the transparency through the protected part. The paper describes different types of electrolytes and compares their shielding effectiveness. The experiment includes a plastic packing, metallic electrodes, liquids, laboratory power supply, a coaxial transmission line with receiving and transmitting conductors, source of electromagnetic radiation, and test receiver. The liquid consists of weak and strong acids and bases, such as acetic acid, sulfuric acid or sodium chloride which have different conductivity. There are also various materials for electrodes affecting the conductivity and consequently, the ability of the electrolyte to eliminate the effects of electromagnetic interference. The kind of electrode affects the amount of received and delivered ions between anode and cathode. Shielding effectiveness of electrolyte depends on more unstable parameters compared to conventional metal materials. Temperature and concentration of solution affect the electrolyte conductivity; thereby, the reproducibility could be problematic. Strong electrolytes initially have a rapid increase in conductivity; however, high amounts of ions cause their interaction and decrease in conductivity. Weak electrolytes have a similar course, but the conductivity achieves many times lower values. Measurement is provided according to the ASTM D4935 methods, and it involves a discontinued inner and flanged outer conductor. Samples and the conductors are isolated from external sources of electromagnetic interference by special absorbent materials. The conclusion describes the contribution of the paper.
\end{abstract}

\section{INTRODUCTION}

Currently, electromagnetic interference has represented an inconvenience for all electronic and electric devices developers. However, this inconvenience can be reduced by electromagnetic shielding. Shielding offers protection for individual components or the entire electronic device, as well as for the human being from dangerous fields. These fields can adversely affect health or vital signs (pacemaker). [1] Shielding is a highly efficient method of electromagnetic protection and is used in a wide range of fields. The conventional way of how to protect the device is a metallic shield which reduces the magnetic and electric field. Fortunately, the progress in material engineering brings the new solution for developers on how to protect the sensitive device. This solution represents intrinsically conducting polymers (ICPs) [2] such as Poly(p-phenylene vinylene), Polyaniline (PANI), polyE-al-polyE or polyE-cu-polyE [3], [4], [5]. Despite these significant scientific successes, electromagnetic interference is a problem in protecting transparent surfaces. Currently, there are not enough transparent materials suitable for this purpose. That is the reason why the authors use unconventional shielding in the form of electrolyte. The expected result of this experiment is to achieve acceptable shielding properties in at least some frequency bands. Although the theory does not support this expectation, the first attempts indicate confirmation of authors' prediction about shielding effectiveness of electrolyte approximately $10 \mathrm{~dB}$.

The paper is divided into four main areas describing the process of the experiment. The first section pays attention to theory background regarding shielding effectiveness. The second section describes the electrolytes used for the tests. The third section involves the process of the experiment, followed by the results. And finally, the contribution of the work is described in the conclusion.

\section{SHIELDING EFFECTIVENESS}

Shielding represents (SE) one of the most critical protections against the negative influence of electrostatic and electromagnetic substances. The publication [6] specifies the shield as a technical construction reducting the field of interfering signals in the defined space. The same publication states the term called as the shielding enclosure, which is a structure protecting the interior against effects of magnetic and electric fields, and at the same time, the structure provides the protection 
to the electronic devices in its vicinity. [6] In addition to the above terms, there is another one characterizing the reducing properties of the material. Shielding effectiveness is defined as a ratio between a signal detected by the receiving antenna without the shield to a signal detected by the antenna with the shield. [6]

Shielding effectiveness is a parameter indicating the ability of the material or barrier limit the passage of the radio frequency signal. The barrier reduces the field strength by means of reflections at the electromagnetic signal transition interfaces and absorption capacities that are indicated by the nature of the material. The electromagnetic wave passing through the surface of the barrier is partially attenuated and divided into smaller waves, one of which continues further into the barrier and the other is reflected off. This process also occurs within the barrier and is described in Figure 1.

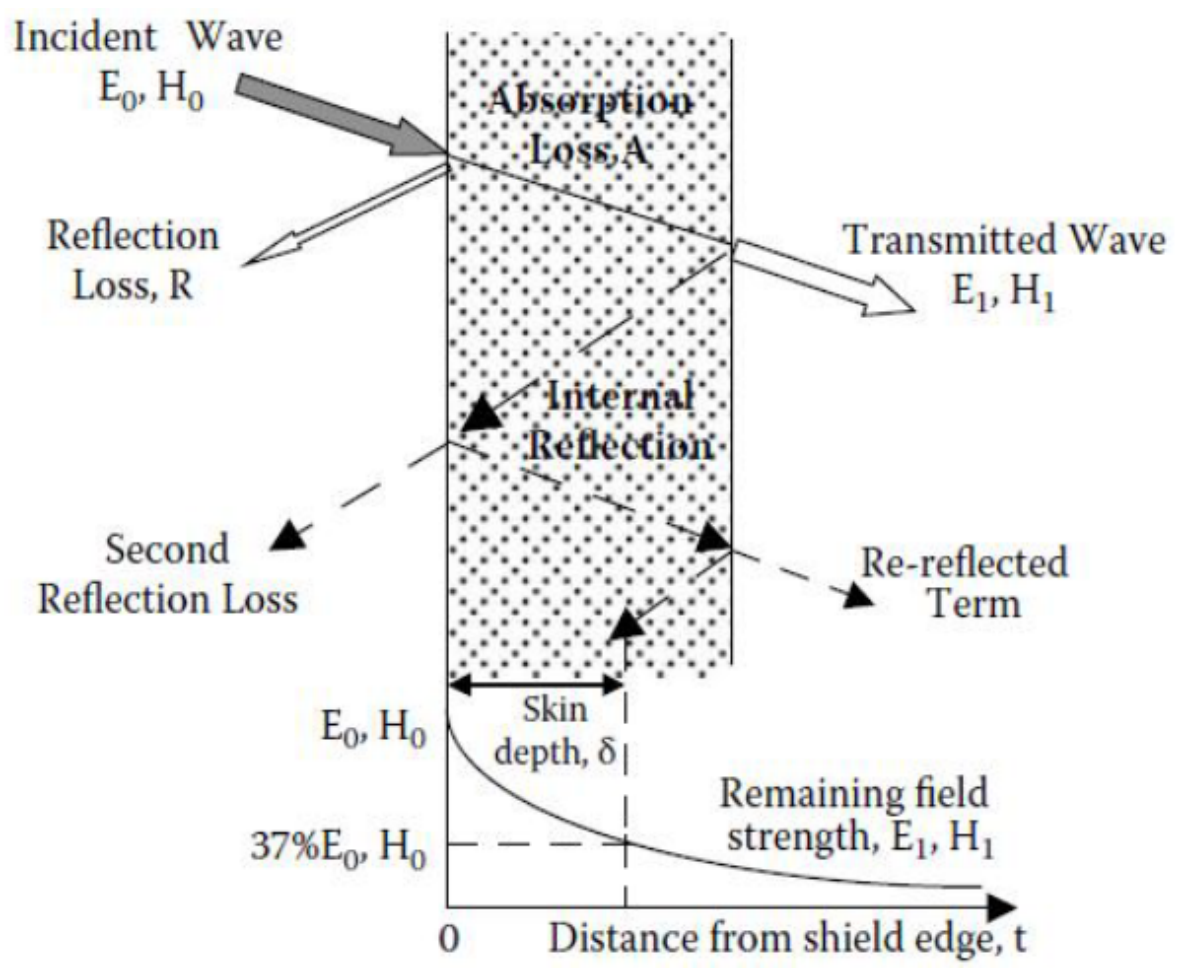

Figure 1: Shielding mechanism [2]

The following equation mathematically expresses the shielding effectiveness:

$$
S E=R+A+M[d B]
$$

where $\mathrm{R}$ is the reflection loss from the barrier surface, $\mathrm{A}$ is the loss through the absorption properties of the barrier, and $\mathrm{M}$ is the multiple reflections within the barrier. Shielding effectiveness describes the differences between the incident wave fell on the surface, and the wave passed through the barrier. Therefore, SE could be expressed in other ways, such as the logarithmic ratio of individual components of the electromagnetic field or difference of absolute power levels.

$$
S E=20 \cdot \log \left|\frac{E_{1}}{E_{2}}\right|=20 \cdot \log \left|\frac{H_{1}}{H_{2}}\right|=L m_{1}-L m_{2}[d B]
$$

The following equations describe the individual losses calculation:

$$
\begin{gathered}
R=20 \cdot \log \left|\frac{Z_{0}}{4 \cdot Z_{M}}\right|[d B] \\
A=8.69 \cdot \frac{t}{\delta}[d B]
\end{gathered}
$$




$$
M=20 \cdot \log \left|1-\left(\frac{Z_{0}-Z_{M}}{Z_{0}+Z_{M}}\right)^{2} \cdot e^{2 \gamma t}\right|[d B]
$$

where $\mathrm{Z}_{0}$ is the characteristic impedance of the free environment, $\mathrm{Z}_{M}$ is the characteristic impedance of the conductive surroundings, $\mathrm{t}$ is the thickness of the barrier, $\delta$ is the depth of penetration of the electromagnetic field into the material, and $\gamma$ is the plane wave propagation constant. The publication [7] claims that the total value of SE can be calculated by substituting equations (3-5) into those described in (1) and (2). Multiple reflections depend on the thickness and conductivity of the material. The equation of SE for the near field is similar except the reflection loss where the $\mathrm{Z}_{0}$ is replaced by the $\mathrm{Z}_{E}$ representing near-field impedance. [7]

$$
Z_{E}=\frac{1}{\omega \epsilon_{0} r}=Z_{0} \cdot \frac{\lambda}{2 \pi r}>>Z_{0}
$$

\section{LIQUID ELECTROLYTE}

The experiment described in the paper consists of the electrolyte with a different kind of electrodes. The research aims to find the most suitable shielding solution; it means experimenting with diverse solutions and materials. An electrolyte is a substance that, when dissolved or melted, breaks down into free-moving charged particles (ions); therefore, acids, bases, and salts are mainly chosen as the electrolyte. The electrolytes use for this experiment include substances such as $\mathrm{NaCl}, \mathrm{H}_{2} \mathrm{SO}_{4}, \mathrm{HCl}$ or $\mathrm{CuSO}_{4} \cdot 5 \mathrm{H}_{2} \mathrm{O}$. DC voltage is applied to the anode and cathode from a laboratory source. The electrode potential is calculated according to the Nernst equation.

$$
E=E^{o}+\frac{R \cdot T}{n \cdot F} \cdot \ln c_{M e^{n+}}
$$

where $\mathrm{R}$ is the molar gas constant, $\mathrm{T}$ is the absolute temperature, $\mathrm{n}$ is the charge of the cation, $\mathrm{F}$ is the Faraday constant and $c_{\mathrm{Me}^{n+}}$ is the concentration of $M e^{n^{+}}$cations in solution.

\section{PROCESS OF EXPERIMENT}

The experiment consists of the plastic packing, metallic electrodes, liquids, laboratory power supply, a coaxial transmission line with receiving and transmitting conductors, source of electromagnetic radiation, and test receiver. The plastic packing includes electrodes with a wire connected to the laboratory power supply and liquid. The power supply has ranged from $0 \mathrm{~V}$ to $15 \mathrm{~V} / 30 \mathrm{~V}$. Location of the electrodes is on opposite sides of the box to create a homogeneous electric field. The electrode material is diverse and included copper, steel, iron, or aluminum. Copper conductors terminate the coaxial transmission line at the one end and by the signal generator or EMI test receiver at the second end. The next variant involves vector network analyzer (VNA), which allows detecting individual components of shielding effectiveness. In other words, VNA displays reflective, multiple reflections, and absorption losses. The plastic packing with liquid is located between the conductors, and the current is applied to the electrodes. During the process of the experiment, the electrodes and the liquid are changed for more accurate results. The frequency range is ranged from $30 \mathrm{MHz}$ to $1 \mathrm{GHz}$.

The experiment is based on the ASTM D4935 method [8], [9], which uses a coaxial line with discontinued inner conductor and a flanged outer conductor. The publication [8] claims that the ASTM provides more accurate SE test than other methods, and it is suitable for analyses of metals, plastics, and materials with high surface resistivity.

The list of equipment used for this experiment:

- ESPI test receiver $(9 \mathrm{kHz}-7 \mathrm{GHz})$

- Rohde\&Schwarz SMF 100A - signal generator (100 kHz - $22 \mathrm{GHz})$

- Rohde\&Schwarz ZND Vector network analyzers (100 kHz - 4.5 GHz)

- BaseTech BT-153 (0 - $15 \mathrm{~V})$

- HQ Power PS3010 (0 - 30 V)

- Agilent U1251A

- $\mathrm{Fe}, \mathrm{Zn}, \mathrm{Al}, \mathrm{Cu}$ electrodes 


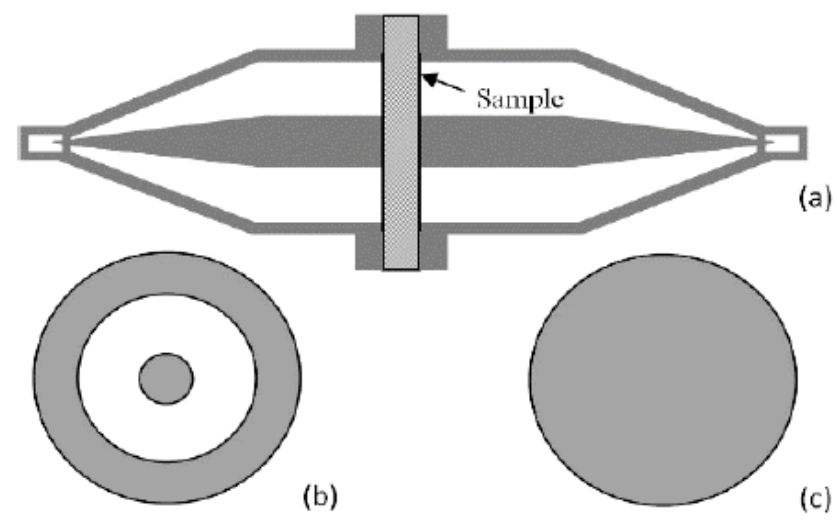

Figure 2: a) ASTM D4935 test device, b) the reference, c) the sample - illustrative figure [8]

\section{RESULTS}

The section describes the results acquired during the experiment, which was carried out according to the specifications given in the measurement process section. The first results are presented in Figure 3 containing shielding effectiveness throughout the tested spectrum. The following table shows the frequencies, including the efficiencies at which the samples achieved the best results. The table offers ranges from worst to best for the selected frequency spectrum. The samples were first tested using the EMI receiver and the EM signal generator. Then the results were verified using the vector analyzer where the parameter of interest was S21 determining the sample throughput.

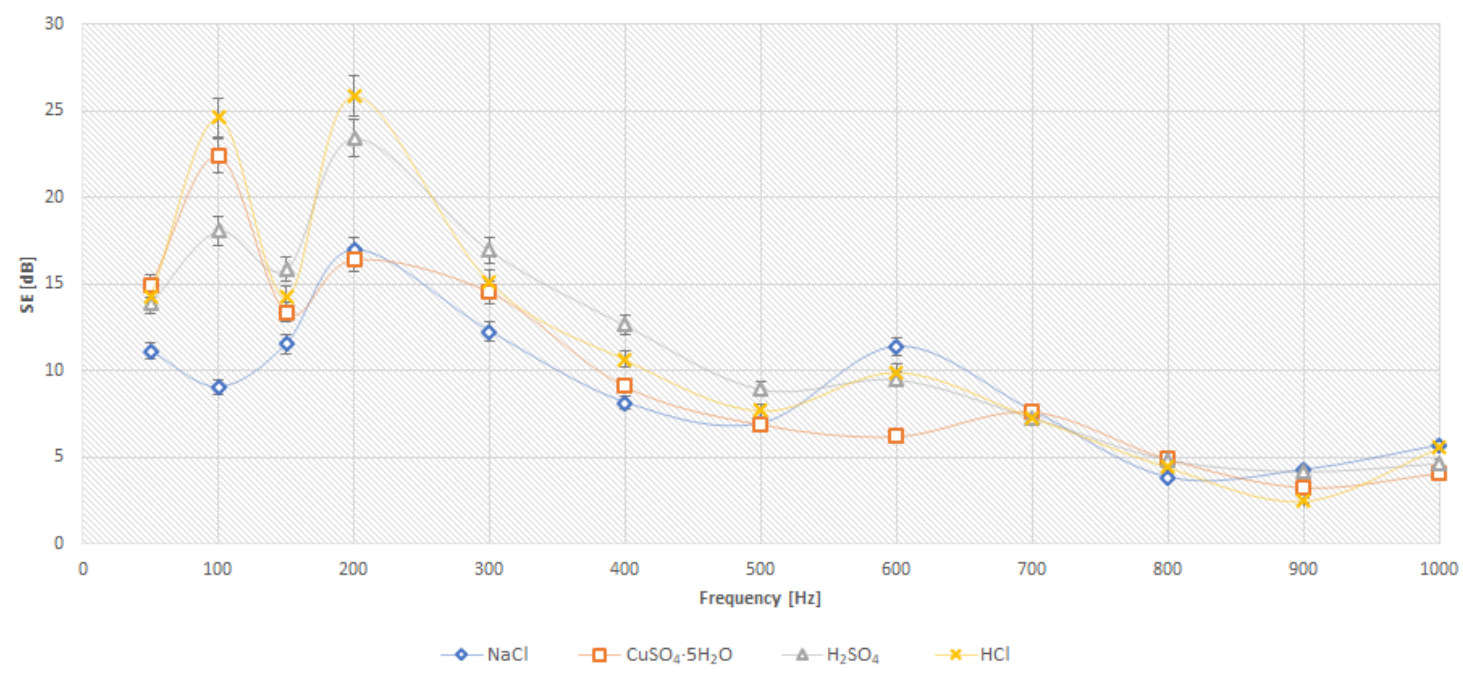

Figure 3: Shielding effectiveness of electrolytes for the frequency range from $50 \mathrm{MHz}$ to $1 \mathrm{GHz}$.

Table 1: The table summarizes the best $\mathrm{SE}$ values of electrolytes concerning frequency.

\begin{tabular}{ccc}
\hline \hline Name of liquid & Frequency range $[\mathrm{MHz}]$ & $\mathrm{SE}[\mathrm{dB}]$ \\
\hline $\mathrm{NaCl}$ & $150-300$ & $12.27-16.96$ \\
$\mathrm{NaCl}$ & 600 & 11.34 \\
$\mathrm{CuSO}_{4} \cdot 5 \mathrm{H}_{2} \mathrm{O}$ & $50-300$ & $13.39-22.4$ \\
$\mathrm{H}_{2} \mathrm{SO}_{4}$ & $50-350$ & $13.91-23.42$ \\
$\mathrm{HCl}$ & $50-300$ & $14.25-25.88$ \\
\hline
\end{tabular}




\section{CONCLUSION}

Paper aims at the shielding effectiveness tests of liquid electrolytes under laboratory conditions. The experiment consists of commercially available conductive substances that can be used to protect transparent surfaces. The SE is tested using a coaxial line terminated by conductors on one side and an EM signal receiver or transmitter on the other side. The experiment confirmed the authors' expectations regarding the low efficiency of electrolytes. However, the shielding efficiency of $\mathrm{HCl}$, $\mathrm{H}_{2} \mathrm{SO}_{4}$, and $\mathrm{CuSO}_{4} \cdot 5 \mathrm{H}_{2} \mathrm{O}$ in the $300 \mathrm{MHz}$ frequency band is surprising. The $\mathrm{SE}$ difference between the fluid and the electrolyte ranged from 2 to $10 \mathrm{~dB}$, with the highest difference being achieved with $\mathrm{HCl}$. The measurement uncertainty is close to $4 \%$. The limiting factor of the experiment was the chemical process as not all suitable materials for electrodes and conductive liquids were tested. The experiment revealed the favorable side shielding properties of the electrolyte, but also confirmed the lack of efficacy of this variant. Future research should focus on removing test limits and using a larger electrode and fluid base.

\section{ACKNOWLEDGMENT}

This work was supported by the Ministry of Education, Youth and Sports of the Czech Republic within the framework of the National Sustainable Development Programme no. LO1303 (MSMT7778/2014) as well as the European Regional Development Fund under the CEBIA-Tech project no. CZ.1.05/2.1.00/03.0089 was supported by research project VI20172019054 "Analysis of software module for real-time immunity assessment in terms of convergent security" supported by the Ministry of the Interior of the Czech Republic in 2017-2019 and the Internal Grant Agency of Tomas Bata University within the project no. IGA/CebiaTech/2018/004.

\section{REFERENCES}

1. Paul, C. R., Introduction to electromagnetic compatibility, John Wiley, New Jersey, 2009.

2. Loya, S. and Habibullakhan, "Analysis of Shielding Effectiveness in the Electric Field and Magnetic Field and Plane Wave for Infinite Sheet Metals," International Journal of Electromagnetics and Applications, Vol. 6, No. 2, 31-41, 2016.

3. Vakente, R., R. De Ruijter, D. Vlasveld, S. Van Der Zwaag and P. Groen, "Setup for EMI Shielding Effectiveness Tests of Electrically Conductive Polymer Composites at Frequencies up to $3.0 \mathrm{GHz}, "$ IEEE Access, Vol. 5, 16665-16675, 2017.

4. Al-Shabib, W., S. W. Lachowicz, "Modelling of intrinsic conducting polymer for Wi-Fi electromagnetic interference shielding," in 2013 Science and Information Conference, London, Great Britain, October 2013, 836-838.

5. Araz, I., "The measurement of shielding effectiveness for small-in-size ferrite-based flat materials," TÜBITAK, Vol. 26, No. 6, 2996-3006, 2018.

6. "IEEE Standard Method for Measuring the Effectiveness of Electromagnetic Shielding Enclosures," in IEEE Std 299-1997, New York, USA, February 2007, 1-52.

7. Svacina, J., Electromagnetic compatibility: principles and notes, Brno University of Technology, Brno, 2001. (in Czech)

8. Morari, C. and I. Balan, "Methods for Determining Shielding Effectiveness of Materials," Electrotehnică, Electronică, Automatică (EEA), Vol. 63, No. 2, 126-136, 2015.

9. Badic, M., M. J. Marinescu, "The failure of coaxial TEM cells ASTM standards methods in H.F. range," in IEEE International Symposium on Electromagnetic Compatibility, Minneapolis, USA, August 2002, 29-34. 